\title{
The Study of Release of Chlorhexidine from Preparations with Modified Thermosensitive Poly-N-isopropylacrylamide Microspheres
}

\author{
Witold Musial, ${ }^{1,2}$ Bojana Voncina, ${ }^{2}$ Janusz Pluta, ${ }^{1}$ and Vanja Kokol ${ }^{2}$ \\ ${ }^{1}$ Chair and Department of Pharmaceutical Technology, Wroclaw Medical University, ul. Szewska 38, 50-139 Wrocław, Poland \\ ${ }^{2}$ Department of Textile Materials and Design, University of Maribor, Smetanova ul. 17, 2000 Maribor, Slovenia
}

Correspondence should be addressed to Witold Musial, witold.musial@gmail.com

Received 11 October 2011; Accepted 20 November 2011

Academic Editor: Doron J. Aframian

Copyright (C) 2012 Witold Musial et al. This is an open access article distributed under the Creative Commons Attribution License, which permits unrestricted use, distribution, and reproduction in any medium, provided the original work is properly cited.

\begin{abstract}
The aim of this study was to investigate and compare the release rates of chlorhexidine (CX) base entrapped in the polymeric beads of modified poly- $N$-isopropylacrylamides (pNIPAMs) at temperatures below and over the volume phase transition temperature (VPTT) of synthesized polymers: pNIPAM-A with terminal anionic groups resulting from potassium persulfate initiator, pNIPAM-B with cationic amidine terminal groups, and pNIPAM-C comprising anionic terminals, but with increased hydrophobicity maintained by the N-tert-butyl functional groups. The preparations, assessed in vitro below the VPTT, release an initial burst of CX at different time periods between 120 and $240 \mathrm{~min}$, followed by a period of $24 \mathrm{~h}$, when the rate of release remains approximately constant, approaching the zero-order kinetics; the release rates for the polymers beads are as follows: pNIPAM$\mathrm{C}>\mathrm{pNIPAM}-\mathrm{B}>\mathrm{pNIPAM}-\mathrm{A}$. The pattern of release rates at temperature over the VPTT is as follows: pNIPAM-C $>$ pNIPAM$\mathrm{A}>\mathrm{pNIPAM}-\mathrm{B}$. In the presence of pNIPAM-C, the duration between the start of the release and the attained minimal inhibitory concentration (MIC) for most of the microbes, in conditions over the VPTT, increased from 60 to 90 min. The release prolongation could be ascribed to some interactions between the practically insoluble CX particle and the hydrophobic functional groups of the polymer.
\end{abstract}

\section{Introduction}

Chlorhexidine (CX), the known and widely applied antimicrobial agent, still is identified as the so-called "gold standard" in many applications, including oral and skin health applications, as well as some other skin and mucosal applications [1]. Researchers are evaluating numerous systems for controlled delivery of CX to the place of the interest, that is, mucosa of oral cavity, or skin surface. Yue et al. prepared, by single emulsion, solvent evaporation technique microparticles of poly(dL-lactic-co-glycolic acid) containing CX-free base, CX digluconate, and their association or inclusion complex with methylated-beta-cyclodextrin and hydroxypropyl-beta-cyclodextrin [2]. Some authors proposed urethane dimethacrylate-triethylene glycol dimethacrylate resin system with some success [3]. Early works included carriers based on acrylic strip [4] and chip of cross-linked collagen [5]. Also natural polymers as xanthan [6] or silk fibroin/gelatin hybrid films [7] were evaluated for loading CX.

The approach to effectively use microspheres for CX delivery to the place of proper activity was evaluated originally by Egbaria and Friedman [8], who evaluated the antibacterial activity of human albumin microspheres containing CX dihydrochloride against bacteria of urinary tract. The microspheres composed of chitosan were soon evaluated for preparation of buccal tablets with CX, acting on the oral mucosa [9]. Wu and Lee [10] applied in theirs microspheres loaded by chlorhexidin modified, that is, acetylated and succinoylated inulin-obtained microspheres were characterized by prolonged release of CX.

Poly- $N$-isopropylacrylamides (pNIPAM) are among the group of most applied macromolecules, characterized by reversible volume-phase transition at ca. $31^{\circ} \mathrm{C}$ [11]. The 
thermosensitive microgel particles may be applied in many medical devices, including drug forms for topical use [12, 13]. With the collapse and expansion of the macromolecule in the aqueous environment, the molecules of the biologically active substance may be released in a controlled manner $[14,15]$. The deswelling process is controlled by diffusion, where the rate of the collapsing of the macromolecule is correlated to the dimensions of the pores in the polymeric matrix [16]. When the VPTT is crossed, the phase transition from one side enables the expelling of the drug from the polymeric environment, but on the other hand the aggregation may be expected, when the microspheres will adhere to the mucosa surface in the oral cavity.

In our previous studies, we investigated release of CX from ionic and nonionic polymer hydrogels, namely, from methylcellulose and modified polyacrylic acid [17]. Some approach was made to evaluate the amounts of CX released from modified poly- $N$-isopropylacrylamide by the conductometry method [18]. The aim of the present work is to evaluate the influence of three different microspheres batches on the release of CX in the conditions below and over the VPTT, applying pharmacopoeia release device.

The pNIPAM microgels, namely, pNIPAM-A, pNIPAM$\mathrm{B}$, and $\mathrm{pNIPAM}-\mathrm{C}$, with different terminal functional groups based on different initiators used in surfactant-free dispersion polymerization (SFDP), were synthesized in previous research [19]. For microgels pNIPAM-A and pNIPAM-C, potassium persulfate was used as an initiator in SFDP, while for pNIPAM-B, 2,2' -azo-bis(2-methylpropionamidine)dihydrochloride was used. The microgel pNIPAM-C was synthesized with the addition of comonomer, N-tert-butyl acrylamide, to increase the hydrophobicity of the received particles due to the experiments performed by Lynch et al. [20] as well as Lindmann et al. [21]. The IR spectra of the freeze-dried polymers obtained from SFEP were compared with that of the pure components to establish the occurrence of polymerization, and some physical assessments like optical microscopy, scanning electron microscopy, VPTT, conductivity, and $\mathrm{pH}$ were carried out to evaluate the products. The acquired release rates and concentrations of CX released from CX-loaded pNIPAM microgels were compared with that obtained from a water dispersion of CX as well as CXloaded methylcellulose (MC) and polyacrylic acid (PA) beads [17].

\section{Materials and Methods}

N-isopropylacrylamide 97\% (Aldrich), N-tert-butyl acrylamide 99\% (Acros Organics), N,N'-methylenebisacrylamide 99\% (Aldrich), potassium persulfate 98\% (BDH Laboratory Suppliers (GPR), and 2,2'-azo-bis(2-methylpropionamidine) dihydrochloride $97 \%$ (Aldrich) were received from commercial and industrial suppliers and used without further purification. Dialysis bag of molecular weight cutoff (MWCO) of 12000-14000 Da was purchased from Visking Medicell International Ltd. Deionized water from the TKA DI 6000 system (Germany) was applied in all the procedures.
2.1. Synthesis of the Microgels. The N-isopropylacrylamide derivative microgel particles were synthesized by SFDP in deionized water at $70^{\circ} \mathrm{C}$, under an inert nitrogen atmosphere due to the procedure reported in former paper [18]. The pNIPAM-A was characterized as a polymer with terminal anionic function groups. The pNIPAM-B was synthesized in the presence of 2,2'-azo-bis(2-methylpropionamidine)dihydrochloride, which resulted in cationic amidine terminal functional groups. The pNIPAM-C was characterized as the polymer with anionic terminal functional groups, but with increased hydrophobicity according to the functional groups introduced during the synthesis. For better evaluation of the polymerization process, the IR spectra assessments were performed to exclude the presence of vinyl groups in the products of the reaction, as well as to confirm the implementation of respective comonomer [18]. Also the other basic data on the characteristics of the microspheres were presented in the previous study, as the VPTT and scanning electron microscopy images of entities obtained through the synthesis.

2.2. Preparations of Microspheres-CX Mixtures. The CXloaded preparations were developed using the polymers pNIPAM-A, pNIPAM-B, pNIPAM-C, and for comparison also MC and PA. The samples were allowed to swell in the $0.5 \%$ dispersions of CX for $48 \mathrm{~h}$ in a water bath at $298 \mathrm{~K}$, under continuous stirring. After $48 \mathrm{~h}$, when the incorporation procedure terminated, the samples were immediately frozen using liquid nitrogen and freeze-dried by MINI LYOTRAP LF/LYO/02/1 with vacuum pump model RV5, in high vacuum mode, at $50 \%$ power setting, with vacuum values in the range of $1 \times 10^{-1}-1 \times 100 \mathrm{mbar}$ (i.e., $1 \times$ $10^{1}-1 \times 10^{2} \mathrm{~Pa}$ ) for $24 \mathrm{~h}$, and finally dispersed in water. The time for complete incorporation was demonstrated by previous consequent spectrophotometric assessments of the filtered samples, which came from the preparations loaded by chlorhexidine in various, increasing time periods. The exponential curve ranged the limit, and the amount of assessed drug did not decrease further after ca. $36 \mathrm{hrs}$. To receive the full load, the margin of $12 \mathrm{hrs}$ was added. Composition of the obtained microgel preparations of CX is given in the attached Table 1.

2.3. Morphology of the Obtained Complexes of Polymer and $C X$. The surface and morphology of the freeze-dried samples were examined using light microscopy (LM). The morphology of the loaded samples was assessed by the optical microscope, Olympus BX51, with oculars $20 \times$ $0.46 \mathrm{~mm}$ and $50 \times 0.80 \mathrm{~mm}$, and recorded using a digital camera, Olympus DP12, U-TVO.5XC-2, by applying direct day light. To obtain more data about the surface of the dry samples, the scanning electron microscope (SEM) was applied, using the FEI QUANTA 200 3D.

2.4. Release Rates. The in vitro release of CX from the solution in water and from the hydrogel preparations across artificial membrane was examined using United States Pharmacopoeia paddle method with the acceptor volume of 
TABLE 1: Composition of investigated preparations.

\begin{tabular}{|c|c|c|c|c|c|c|c|}
\hline Components preparation & $\mathrm{CX}(\mathrm{mg})$ & pNIPAM-A (mg) & pNIPAM-B (mg) & pNIPAM-C (mg) & $\mathrm{MC}(\mathrm{mg})$ & $\mathrm{PA}(\mathrm{mg})$ & Water $(\mathrm{g})$ \\
\hline pNIPAM-A-CX & 45 & 90 & - & - & - & - & 20 \\
\hline pNIPAM-B-CX & 45 & - & 90 & - & - & - & 20 \\
\hline pNIPAM-C-CX & 45 & - & - & 90 & - & - & 20 \\
\hline MC-CX & 45 & - & - & - & 90 & - & 20 \\
\hline PA-CX & 45 & - & - & - & - & 90 & 20 \\
\hline $\mathrm{H}_{2} \mathrm{O}-\mathrm{CX}$ & 45 & - & - & - & - & - & 20 \\
\hline
\end{tabular}

pNIPAM-A-CX, pNIPAM-B-CX, pNIPAM-C-CX: preparations of respective polymers detailed in the text with chlorhexidine, MC-CX: preparation of methylcellulose and chlorhexidine, PA-CX: preparation of polyacrylic acid and chlorhexidine, and $\mathrm{H}_{2} \mathrm{O}-\mathrm{CX}$ : aqueous dispersion of chlorhexidine.

$900 \mathrm{~mL}$ and a defined diffusional area of $64 \mathrm{~cm}^{2}$. Water was used as the acceptor phase, and it was adjusted to a $\mathrm{pH}$ of 5.5 by the addition of small quantities of $0.1 \mathrm{M} \mathrm{HCl}$, to maintain the $\mathrm{pH}$ close to the physiological $\mathrm{pH}$ of the skin surface. After preparation, all the samples, that is, CX dispersion and loaded hydrogels, were placed in a thermostated water bath, at 22 and $37^{\circ} \mathrm{C}$ for $24 \mathrm{~h}$. About $20 \mathrm{~mL}$ of the sample, either of the solution of $\mathrm{CX}$ or a hydrogel containing the drug, was placed in the donor compartment. Six experiments were conducted using these systems for a $24 \mathrm{~h}$ period. About $1 \mathrm{~mL}$ of receptor phase was taken as a sample and replaced with fresh prethermostated acceptor phase. The vessels were silanized using dichlorodimethyl silane to minimize CX adsorption on the glass. The samples were taken every $10 \mathrm{~min}$ for the first $100 \mathrm{~min}$, then, every $30 \mathrm{~min}$ for the next $2 \mathrm{~h}$, and, subsequently, every $1 \mathrm{~h}$ for up to $12 \mathrm{~h}$, to achieve 20 measurement points for this time period. The CX in the eluent was assessed using the UV spectrophotometric method. The percentage extinction coefficient $(\mathrm{a} 1 \%, 1 \mathrm{~cm})$ in $0.01 \mathrm{~mol} / \mathrm{L}$ $\mathrm{HCl}$ solution was $224.12(P>0.999)$ at $250 \mathrm{~nm}$, and the method gave a linear response over a concentration range of $1-20 \mu \mathrm{g} / \mathrm{mL}$ [22]. The UV spectrophotometer, TECAN Infinite 200, with 96 wells plates, Greiner 96 flat-bottom transparent polystyrol plates, was used for the determination of CX concentrations in the studied samples.

\section{Results and Discussion}

The polymeric microgels obtained in the process of SFDP were prepared using known method proposed and evaluated, that is, by Pelton [23] as well as Saunders and Vincent [24]. Consequent IR assessments confirmed the reduction of the double bonds in the monomer molecule [25], demonstrating that the polymer was obtained [26]. The VPTT was observed in the turbidity measurements, and, hence, all the synthesized products were considered as thermosensitive [27]. In the present research, we confirmed the detailed evaluations performed earlier-the VPTT for PNIPAM-A and pNIPAM-B was around $34^{\circ} \mathrm{C}$, whereas, for pNIPAM$\mathrm{C}$, it was observed at $32^{\circ}$. The conductivity measurements revealed complete purification of obtained material from the initiator or comonomer remains.

Both LM and SEM observations of freeze-dried material revealed hydrogel-like structure in the case of CXpNIPAM-A compositions, whereas for CX-pNIPAM-B and
CX-pNIPAM-C compositions, we observed microspheres of diameter between 0.5 and $5 \mu \mathrm{m}$ (Figures 1 and 2). As it is evident from the LM microphotography, the spheres tended to aggregate, however, in the observation field, there were no particles actually aggregated. The SEM photograph, panel A (Figure 1), gives impression of some artifacts or deposited CX on the surface of the fiber-like structures. On the panel B of Figure 2, the obtained microgels have some observable empty areas, between planar surfaces of poly- $N$-isopropylacrylamide, very characteristic for this kind of material, possibly with some CX binded. This both interesting issues, dealing with suspected specific CX deposition on/in microgels, will be evaluated in next study. The hydrophobized particles had rather smooth surface in the SEM observations, with no observable artifacts, for example, CX crystals.

The $\mathrm{CX}$ preparations, assessed in vitro at $22^{\circ} \mathrm{C}$, that is, below the VPTT for the studied microgels, released an initial burst of CX at different time periods between 120 and $240 \mathrm{~min}$, followed by a period of ca. $24 \mathrm{~h}$, in which the rate of release was approximately constant, approaching the zero-order kinetics as it is depicted in Figure 3. To determine whether the release rate decreased exponentially, the logarithms of the CX concentrations were plotted against time, which produced straight lines, as shown in Figure 4. The linear regression coefficients given in Table 2 were over 0.9584 , indicating an extremely close fit of the values to the line and supporting the premise that the release rate decreased exponentially during the assay.

Depending on the preparations, the rates fell by approximately up to 10 times in a period of $24 \mathrm{~h}$. During that period, the concentration of CX attained in each sample level of $>20 \mu \mathrm{g} / \mathrm{mL}$, which is well above the minimal inhibitory concentration (MIC) for most of the microbes (Figure 3). The release rate was the highest in the case of aqueous suspension of the $\mathrm{CX}$ base. The release rates for the polymers beads were as follows: pNIPAM-C $>$ pNIPAM-B $>$ pNIPAM$\mathrm{A}>\mathrm{MC}>\mathrm{PA}$. Furthermore, the prolongation of CX release was ca. $30-50 \mathrm{~min}$. The release of CX in the presence of pNIPAM can be divided into two stages (Table 3), and the transition point for PNIPAM-A and PNIPAM-B was observed at $90 \mathrm{~min}$, whereas, for pNIPAM-C, the apparent release rate decreased significantly after $130 \mathrm{~min}$.

Additionally, the first-stage release rate for pNIPAM$\mathrm{C}$ was significantly higher than those for PNIPAM-A and 


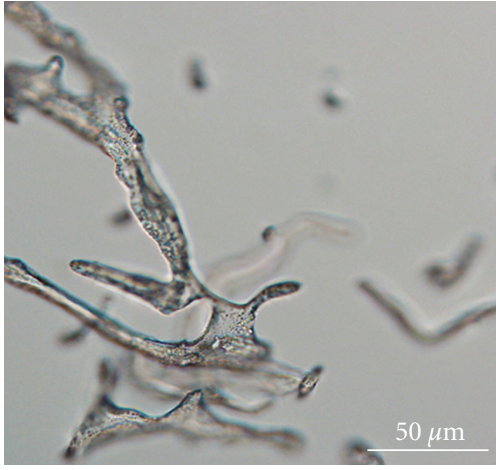

(a)

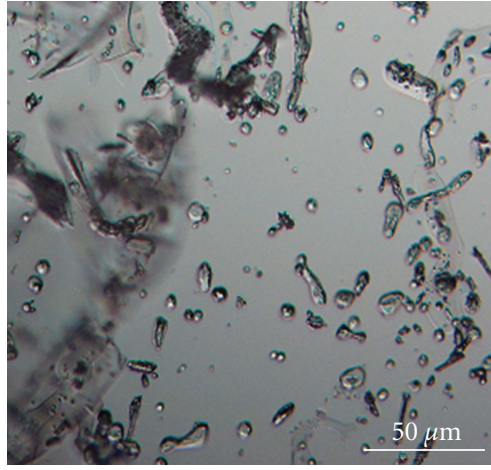

(b)

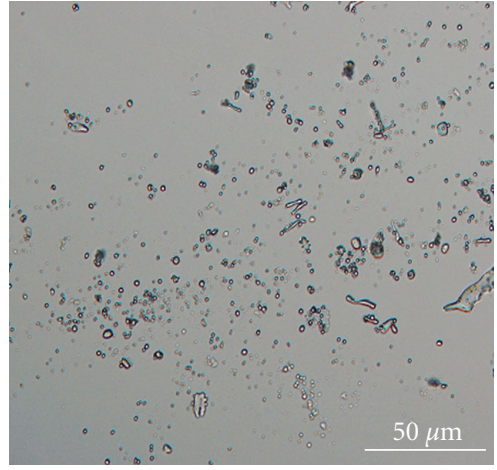

(c)

FIgURE 1: Morphology of obtained polymers; the light microscopy images: (a) pNIPAM-A, (b) pNIPAM-B, (c) pNIPAM-C.

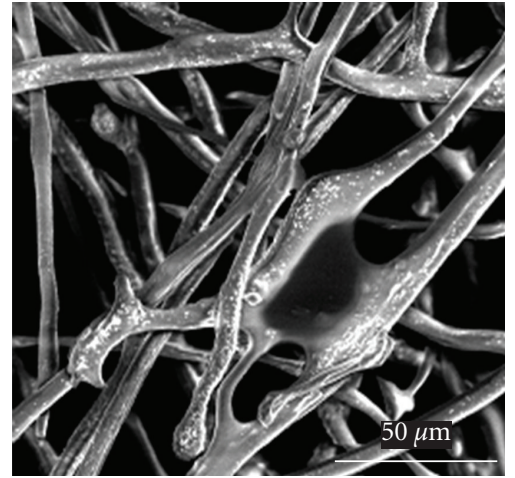

(a)

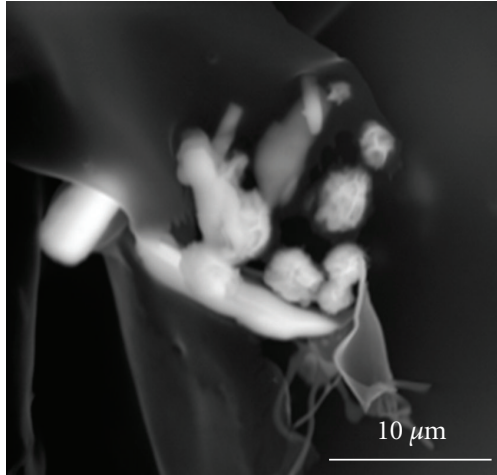

(b)

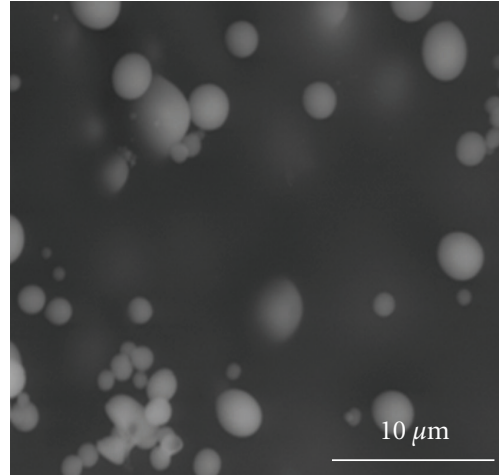

(c)

FIGURE 2: Morphology of obtained polymers; the scanning electron microscopy images: (a) pNIPAM-A, (b) pNIPAM-B, (c) pNIPAM-C.

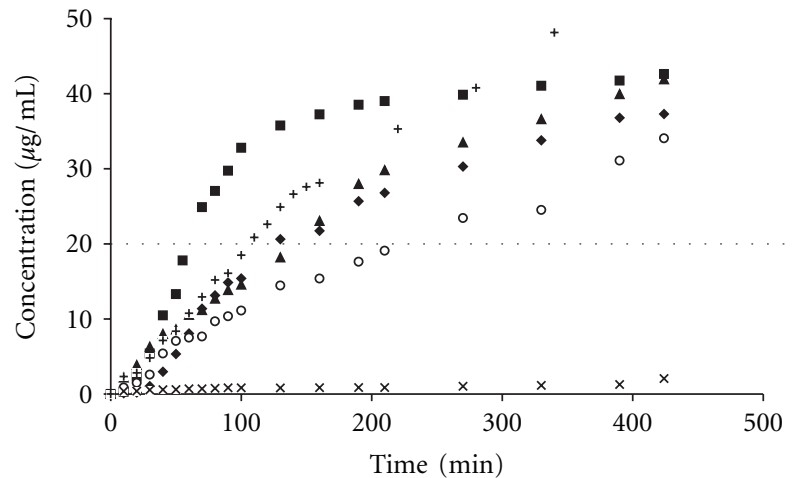

Figure 3: Influence of the type of polymer in the donor compartment on the CX concentration in the acceptor medium at $22^{\circ} \mathrm{C}$. The release from respective preparations was depicted: pNIPAMA-CX $(\downarrow)$, pNIPAM-B-CX ( $)$, pNIPAM-C-CX (ם), MC-CX (o), $\mathrm{PA}-\mathrm{CX}(\times)$, and aqueous dispersion of $\mathrm{CX}(+)$, data from repeated six experiments. The dashed straight line, parallel to the $x$-axis, represents the MIC of CX for Staphylococcus mutans.

pNIPAM-B. Also, the release curves for pNIPAM-A and pNIPAM-B did not show much difference in group. For comparison, the respective data of the release rates for MC and PA are presented in Table 3, although the VPTT

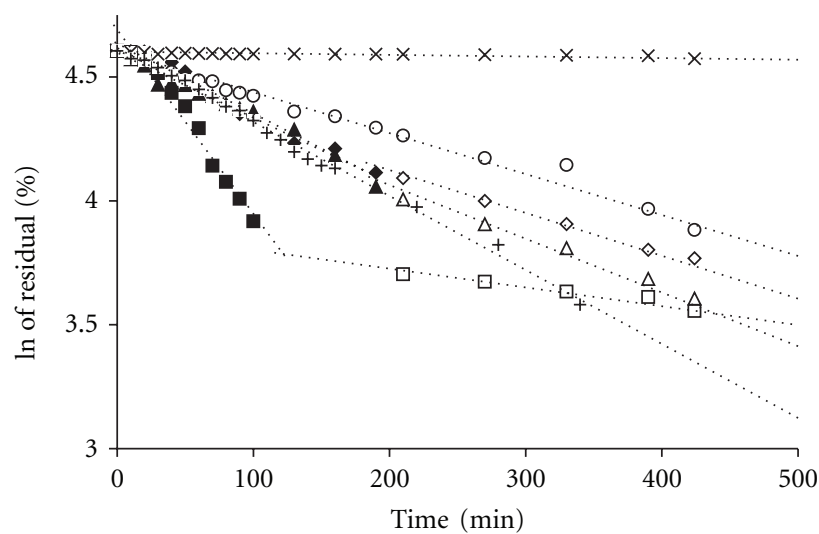

FIGURE 4: Influence of the type of polymer on the release kinetics of CX from the polymeric bead at $22^{\circ} \mathrm{C}$. The release from respective preparations was depicted as follows: pNIPAM-A-CX for first stage $(\diamond)$ and second stage $(\diamond)$, pNIPAM-B-CX for first stage $(\Delta)$ and second stage $(\triangle)$, pNIPAM-C-CX for first stage $(\boldsymbol{\square})$ and second stage $(\square)$, MC-CX (०), PA-CX $(\times)$, and aqueous dispersion of CX $(+)$, data from repeated six experiments.

was not assessed for these polymers. For a specific time period, the release of CX at temperature over VPTT was different than that at temperature below VPTT (Figure 5). 
TABLE 2: Linear regression coefficients for the assessed release of CX from the polymeric preparations, assuming first-order process.

\begin{tabular}{|c|c|c|c|c|}
\hline \multirow{2}{*}{$\begin{array}{l}\text { Release rates } \\
\text { Preparation }\end{array}$} & \multicolumn{2}{|c|}{$\begin{array}{l}\text { Release rates assessed at } \\
\qquad 22^{\circ} \mathrm{C}\left(\mathrm{min}^{-1}\right)\end{array}$} & \multicolumn{2}{|c|}{$\begin{array}{l}\text { Release rates assessed at } \\
\qquad 37^{\circ} \mathrm{C}\left(\mathrm{min}^{-1}\right)\end{array}$} \\
\hline & 1st stage & 2nd stage & 1st stage & 2nd stage \\
\hline pNIPAM-A-CX & 0.9737 & 0.9864 & 0.9897 & 0.9675 \\
\hline pNIPAM-B-CX & 0.9780 & 0.9783 & 0.9941 & 0.9967 \\
\hline pNIPAM-C-CX & 0.9670 & 0.9584 & 0.9823 & 0.9960 \\
\hline MC-CX & 0.9884 & - & 0.9838 & - \\
\hline PA-CX & 0.8585 & - & 0.6922 & - \\
\hline $\mathrm{H}_{2} \mathrm{O}-\mathrm{CX}$ & 0.9949 & - & 0.9761 & - \\
\hline
\end{tabular}

pNIPAM-A-CX, pNIPAM-B-CX, pNIPAM-C-CX: preparations of respective polymers detailed in the text with chlorhexidine, MC-CX: preparation of methylcellulose and chlorhexidine, PA-CX: preparation of polyacrylic acid and chlorhexidine, and $\mathrm{H}_{2} \mathrm{O}-\mathrm{CX}$ : aqueous dispersion of chlorhexidine, data from repeated six experiments.

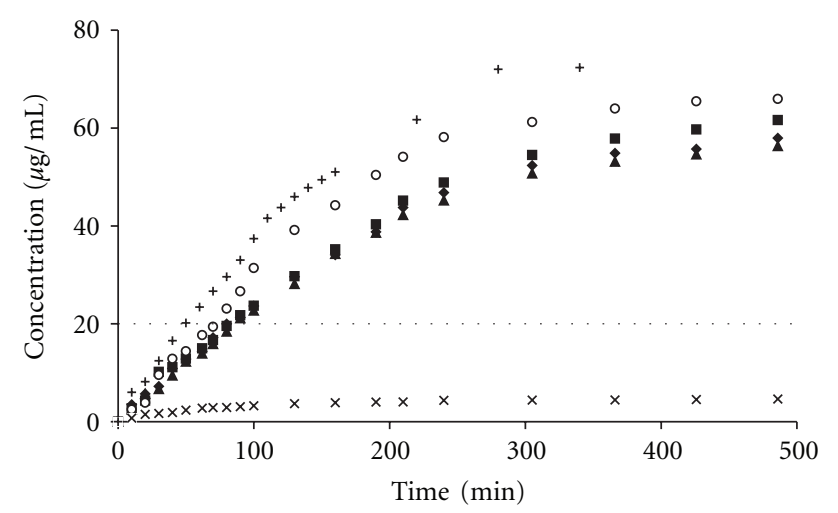

Figure 5: Influence of the type of polymer in the donor compartment on the CX concentration in the acceptor medium at $37^{\circ} \mathrm{C}$. The release from respective preparations was depicted: pNIPAMA-CX $(\downarrow)$, pNIPAM-B-CX ( $\mathbf{\Delta})$, pNIPAM-C-CX (ם), MC-CX (०), PA-CX $(X)$, and aqueous dispersion of $C X(+)$, data from repeated six experiments. The dashed straight line, parallel to the $x$-axis, represents the MIC of CX for Staphylococcus mutans.

The acquired concentrations exceeded the known mean value of MIC of ca. $20 \mu \mathrm{g} / \mathrm{mL}$. Furthermore, the logarithms of the CX concentrations were plotted against time, as described earlier (Figure 6). The sequence of release rates for the assessed polymers can be presented as follows: MC $>$ pNIPAM-C $>$ pNIPAM-A $>$ pNIPAM-B $>$ PA. The highest release rate was observed for the aqueous suspension of the CX base. PA was the agent, which significantly influenced the release of CX. From the data presented in Table 3, it can be observed that the concentration of CX released from the PA preparation was extremely low.

To determine whether the synthesized polymers would influence the release kinetics of CX, an in vitro model was used, in which the samples of CX with polymer were exposed to a buffer under conditions recognized by the European Pharmacopoeia and the United States Pharmacopoeia as proper for the comparison of semisolid-drug forms applied topically. It would be unlikely for the release rates of CX and the concentrations observed in the acceptor fluid to

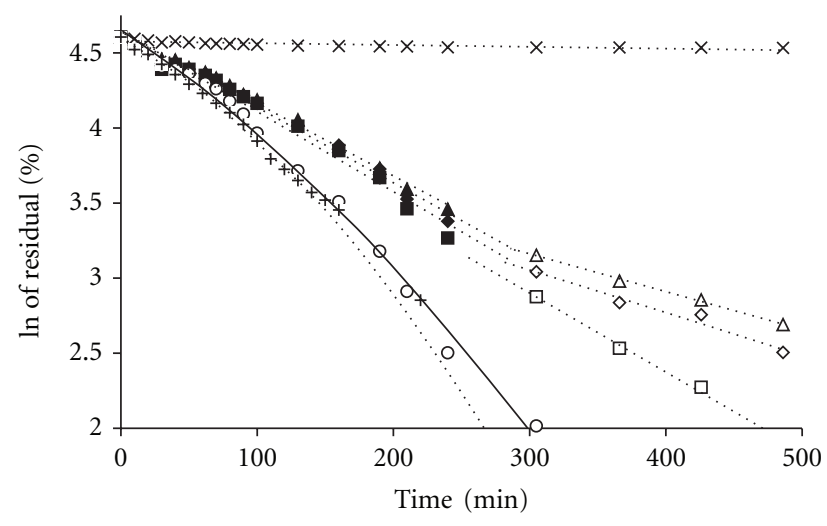

FIGURE 6: Influence of the type of polymer on the release kinetics of $\mathrm{CX}$ from the polymeric bead at $37^{\circ} \mathrm{C}$. The release from respective preparations was depicted as follows: pNIPAM-A-CX for first stage $(\diamond)$ and second stage $(\diamond)$, pNIPAM-B-CX for first stage $(\boldsymbol{\Delta})$ and second stage $(\triangle)$, pNIPAM-C-CX for first stage $(\square)$ and second stage $(\square)$, MC-CX (०), PA-CX $(\times)$, and aqueous dispersion of CX $(+)$, data from repeated six experiments.

be in exact agreement with those on the skin surface, where considerable variation in these parameters can be expected with regard to the differences in the location of the dermis, exposure to surface lipids, washing, and other effects observed on the skin surface. Nevertheless, the model system demonstrated that the characteristics of the kinetics of release differ among the applied polymers. Release systems, like those described in this study, in which a drug is dissolved or dispersed in a polymer vehicle, can be classified as diffusional matrix systems. They usually exhibit first-order release kinetics, in which the rate of release diminishes exponentially with time, in response to the decreasing concentrations of the drug in the polymeric matrix. However, the zero-order release in numerous cases is considered to be the ideal system for drug administration. That case is considered to occur within a matrix system, when the drug is present in the core of the matrix in a highly saturated state. Consequently, the diffusion of the drug from the matrix is rate limiting [28].

The data from the kinetics experiment were also employed for the evaluation of the maximum dose of CX that might be absorbed over an $8 \mathrm{~h}$ period by a patient receiving a topical treatment. The received concentration in the acceptor fluid was up to $13 \mathrm{mg} / \mathrm{mL}$ in the first hour, equivalent to a release of an estimated $1300 \mathrm{mg}$ of the drug for the same period at a temperature over the VPTT, under skin conditions. This is far below the daily dose of $2000 \mathrm{mg}$ of CX that humans are capable of ingesting without producing any adverse effects [29]. However, at temperature below VPTT, the values were not higher than $10 \mathrm{mg}$ for pNIPAM$B$ and PNIPAM-C and reached almost $20 \mathrm{mg}$ for pNIPAMA. In this case, the evaluated concentration of CX on the skin reached the dose of $2000 \mathrm{mg}$. Thus, the differences in the release condition should be taken into account when thermosensitive particles are employed, such as drug carriers, for the topical and dermal applications as well.

The effective concentration of the drug on the skin surface was also evaluated in terms of the time period 
TABLE 3: Release rates of CX from polymeric preparations.

\begin{tabular}{|c|c|c|c|c|c|c|c|c|}
\hline \multirow{2}{*}{ Polymer } & \multicolumn{4}{|c|}{ Release rates assessed at $22^{\circ} \mathrm{C}\left(\mathrm{min}^{-1}\right)$} & \multicolumn{4}{|c|}{ Release rates assessed at $37^{\circ} \mathrm{C}\left(\mathrm{min}^{-1}\right)$} \\
\hline & 1st stage & $\mathrm{SD}$ & 2nd stage & SD & 1st stage & SD & 2nd stage & SD \\
\hline pNIPAM-A-CX & $2.85 \times 10^{-3}$ & $0.01 \times 10^{-3}$ & $1.74 \times 10^{-3}$ & $0.02 \times 10^{-3}$ & $4.98 \times 10^{-3}$ & $0.02 \times 10^{-3}$ & $2.80 \times 10^{-3}$ & $0.26 \times 10^{-3}$ \\
\hline pNIPAM-B-CX & $2.62 \times 10^{-3}$ & $0.02 \times 10^{-3}$ & $2.15 \times 10^{-3}$ & $0.05 \times 10^{-3}$ & $4.79 \times 10^{-3}$ & $0.04 \times 10^{-3}$ & $2.50 \times 10^{-3}$ & $0.18 \times 10^{-3}$ \\
\hline pNIPAM-C-CX & $7.43 \times 10^{-3}$ & $0.04 \times 10^{-3}$ & $7.57 \times 10^{-4}$ & $0.40 \times 10^{-4}$ & $5.37 \times 10^{-3}$ & $0.04 \times 10^{-3}$ & $5.26 \times 10^{-3}$ & $0.39 \times 10^{-3}$ \\
\hline MC-CX & $1.66 \times 10^{-3}$ & $0.03 \times 10^{-3}$ & - & - & $1.22 \times 10^{-2}$ & $0.01 \times 10^{-2}$ & - & - \\
\hline PA-CX & $3.60 \times 10^{-5}$ & $0.04 \times 10^{-5}$ & - & - & $4.09 \times 10^{-5}$ & $0.05 \times 10^{-5}$ & - & - \\
\hline $\mathrm{H}_{2} \mathrm{O}-\mathrm{CX}$ & $3.29 \times 10^{-3}$ & $0.07 \times 10^{-3}$ & - & - & $1.06 \times 10^{-2}$ & $0.03 \times 10^{-2}$ & - & - \\
\hline
\end{tabular}

pNIPAM-A-CX, pNIPAM-B-CX, pNIPAM-C-CX: preparations of respective polymers detailed in the text with chlorhexidine, MC-CX: preparation of methylcellulose and chlorhexidine, PA-CX: preparation of polyacrylic acid and chlorhexidine, and $\mathrm{H}_{2} \mathrm{O}-\mathrm{CX}$ : aqueous dispersion of chlorhexidine, data from repeated six experiments.

TABLE 4: Assessed effective times*.

\begin{tabular}{|c|c|c|c|}
\hline Preparation & $\begin{array}{l}\text { Effective time at the temperature } \\
\text { of } 22^{\circ} \mathrm{C}(\mathrm{min})\end{array}$ & $\begin{array}{l}\text { Effective time at the temperature } \\
\qquad \text { of } 37^{\circ} \mathrm{C}(\mathrm{min})\end{array}$ & $\begin{array}{l}\text { The decrease of time at which the MIC is } \\
\text { acquired with the increase in the } \\
\text { temperature in the range of } 15^{\circ} \mathrm{C}(\mathrm{min})\end{array}$ \\
\hline pNIPAM-A-CX & 130 & 80 & 50 \\
\hline pNIPAM-B-CX & 145 & 80 & 65 \\
\hline pNIPAM-C-CX & 60 & 90 & -30 \\
\hline $\mathrm{MC}$ & 210 & 70 & 140 \\
\hline CX & 110 & 50 & 60 \\
\hline PA & $\mathrm{n} / \mathrm{a}$ & $\mathrm{n} / \mathrm{a}$ & $\mathrm{n} / \mathrm{a}$ \\
\hline
\end{tabular}

* Effective time was recognized as the period from the start of the release to the moment when MIC for CX was observed, n/a: not assessed.

after which the concentration of MIC could be acquired. In general, the presence of polymers resulted in the decrease of release rate, with the extreme case of PA. When the temperature increased, the MIC in the acceptor fluid was observed in advance, and the MC preparation was the most significant case. On the other hand, the increase in the release rate and the corresponding decrease in the effective time can be elucidated in terms of the decrease in viscosity with respect to the increase in temperature. Nevertheless, in the presence of pNIPAM-C microgel, the effective time, that is, the duration since the start of the release to the moment when the MIC is reached, in thermal conditions over the VPTT, increased from 60 to $90 \mathrm{~min}$. The pNIPAM-C polymer was characterized as a hydrophobized macromolecule, with butyl acrylate functional group-thus, the release prolongation could be attributed to some interactions between the insoluble CX molecule and the hydrophobic functional groups of the polymer. The assessed effective times are presented in Table 4.

The MIC of CX for Streptococcus specia, one of the main pathogens in the respiratory tract, is in the range of $0.25-64 \mu \mathrm{g} / \mathrm{mL}$, that is, $0.25-64 \mathrm{mg} / \mathrm{L}$ [30-32]. The proposed polymer systems for the release of active substance enable the achievement of direct bactericidal and bacteriostatic levels of CX in the application area after ca. $60-130 \mathrm{~min}$, in the case of PNIPAM polymers at temperature below the VPTT. However, when the temperature is over the VPTT value for the assessed synthesized polymers, the antibacterial effect is observed at $80-90 \mathrm{~min}$. The release of the CX from the polymeric preparations is observed to intensify simultaneously with the increase in the temperature during the release process. However, the only exception was pNIPAM-C. In the presence of this polymer, the effective time, that is, time needed to observe the MIC in the acceptor compartment, increased at the temperature over the VPTT. This can be owing to the effective embedding of the CX in the microsphere structure-one of the factors may be the lipophilic interaction of the polymer tertiarybutyl chains with the CX molecules. The future development of the $\mathrm{N}$-isopropyl acrylamide microgels as drug carriers is supported by reported cytocompatibility of the pNIPAM nanoparticles [33]. The obtained data may be valuable for the development of new drug forms for controlled delivery of active substances onto the skin at different environmental temperatures, where the skin surface temperature could vary in the range from $14^{\circ} \mathrm{C}$ up to $42^{\circ} \mathrm{C}$. Therefore, the therapeutic activity of the locally applied drug depends on both the thermodynamic activity of the active molecule compound and the pharmaceutical system, which enables the release of the molecule, as well as on the vasoconstrictive activity of the skin blood vessels. However, the release of the drug might be different when a patient is suffering from fever, when compared with the healthy subject. Furthermore, cryotherapy might also influence the release of the drug applied onto the skin.

\section{Conclusions}

The preparations evaluated in this study could be employed as CX carriers to achieve effective drug concentrations at 
different skin surface temperatures. The proposed polymer systems for the release of active substance enable the achievement of direct bactericidal and bacteriostatic levels of CX in the application area after ca. $60-130 \mathrm{~min}$, in the case of PNIPAM polymers at temperature below the VPTT. The antibacterial effect is observed at $80-90 \mathrm{~min}$ when the temperature is over the VPTT value. Also, the data obtained for the in vitro preliminary selection of the thermosensitive polymers could be used for the further in vivo assays. This class of thermosensitive polymers can be further developed to achieve controlled release of the drug at different thermal conditions of the body.

\section{Acknowledgments}

This research was cofinanced by a Marie Curie Transfer of Knowledge Fellowship of the European Community 6th Frame Program under Contract no. MTKD-CT-2005029540-POLYSURF, at the University of Maribor. Authors are grateful to Professor Brian Vincent from the University of Bristol for the valuable comments and to Mr. Tonica Boncina from the University of Maribor for the assistance in SEM measurements.

\section{References}

[1] A. V. Ankola, M. Hebbal, and M. Mocherla, "A review of efficacy of various modes of chlorhexidine delivery," Journal of Oral Biosciences, vol. 50, no. 4, pp. 239-242, 2008.

[2] I. C. Yue, J. Poff, M. E. Cortés et al., "A novel polymeric chlorhexidine delivery device for the treatment of periodontal disease," Biomaterials, vol. 25, no. 17, pp. 3743-3750, 2004.

[3] K. J. Anusavice, N. Z. Zhang, and C. Shen, "Controlled release of chlorhexidine from UDMA-TEGDMA resin," Journal of Dental Research, vol. 85, no. 10, pp. 950-954, 2006.

[4] M. Addy, L. Rawle, R. Handley, H. N. Newman, and J. F. Coventry, "The development and in vitro evaluation of acrylic strips and dialysis tubing for local drug delivery," Journal of Periodontology, vol. 53, no. 11, pp. 693-699, 1982.

[5] M. K. Jeffcoat, K. S. Bray, S. G. Ciancio et al., "Adjunctive use of a subgingival controlled-release chlorhexidine chip reduces probing depth and improves attachment level compared with scaling and root planing alone," Journal of Periodontology, vol. 69, no. 9, pp. 989-997, 1998.

[6] M. Paolantonio, S. D’Ercolev, A. Pilloni et al., "Clinical, microbiologic, and biochemical effects of subgingival administration of a xanthan-based chlorhexidine gel in the treatment of periodontitis: a randomized multicenter trial," Journal of Periodontology, vol. 80, no. 9, pp. 1479-1492, 2009.

[7] S. Wilaiwan, S. Yaowalak, B. Yodthong, and S. Prasong, "Silk fibroin/gelatin hybrid films for medical applications: study on chlorhexidine diacetate," Journal of Biological Sciences, vol. 10, no. 5, pp. 455-459, 2010.

[8] K. Egbaria and M. Friedman, "Sustained in vitro activity of human albumin microspheres containing chlorhexidine dihydrochloride against bacteria from cultures of organisms that cause urinary tract infections," Antimicrobial Agents and Chemotherapy, vol. 34, no. 11, pp. 2118-2121, 1990.

[9] P. Giunchedi, C. Juliano, E. Gavini, M. Cossu, and M. Sorrenti, "Formulation and in vivo evaluation of chlorhexidine buccal tablets prepared using drug-loaded chitosan microspheres,"
European Journal of Pharmaceutics and Biopharmaceutics, vol. 53, no. 2, pp. 233-239, 2002.

[10] X. Y. Wu and P. I. Lee, "Preparation and characterization of inulin ester microspheres as drug carriers," Journal of Applied Polymer Science, vol. 77, no. 4, pp. 833-840, 2000.

[11] B. R. Saunders, H. M. Crowther, G. E. Morris, S. J. Mears, T. Cosgrove, and B. Vincent, "Factors affecting the swelling of poly(N-isopropylacrylamide) microgel particles: fundamental and commercial implications," Colloids and Surfaces A, vol. 149, no. 1-3, pp. 57-64, 1999.

[12] M. T. Moran, W. M. Carroll, I. Selezneva, A. Gorelov, and Y. Rochev, "Cell growth and detachment from protein-coated PNIPAAm-based copolymers," Journal of Biomedical Materials Research A, vol. 81, no. 4, pp. 870-876, 2007.

[13] I. Ankareddi and C. S. Brazel, "Synthesis and characterization of grafted thermosensitive hydrogels for heating activated controlled release," International Journal of Pharmaceutics, vol. 336, no. 2, pp. 241-247, 2007.

[14] J. R. Retama, B. Frick, T. Seydel, M. Stamm, A. F. Barbero, and E. L. Cabarcos, "Polymer chain dynamics of core-shell thermosensitive microgels," Macromolecules, vol. 41, no. 13, pp. 4739-4745, 2008.

[15] D. Gan and L. A. Lyon, "Synthesis and protein adsorption resistance of PEG-modified poly(N-isopropylacrylamide) core/shell microgels," Macromolecules, vol. 35, no. 26, pp. 9634-9639, 2002.

[16] F. Y. Pong, M. Lee, J. R. Bell, and N. T. Flynn, "Thermoresponsive behavior of poly(N-isopropylacrylamide) hydrogels containing gold nanostructures," Langmuir, vol. 22, no. 8, pp. 3851-3857, 2006.

[17] W. Musial, V. Kokol, and B. Voncina, "Deposition and release of chlorhexidine from non-ionic and anionic polymer matrices," Chemical Papers, vol. 64, no. 3, pp. 346-353, 2010.

[18] W. Musial, V. Kokol, and B. Voncina, "The preliminary assessment of chlorhexidine and lidocaine release from preparations of anionic polymer, evaluated by the conductivity measurements," Polimery w Medycynie, vol. 39, no. 2, pp. 3$15,2009$.

[19] W. Musial, B. Vincent, A. Szumny, and B. Voncina, "Morphological characteristics of modified freeze-dried poly(N-isopropylacrylamide) microspheres studied by optical microscopy, SEM, and DLS," Chemical Papers, vol. 64, no. 5, pp. 602-612, 2010.

[20] I. Lynch, I. A. Blute, B. Zhmud et al., "Correlation of the adhesive properties of cells to $\mathrm{N}$-isopropylacrylamide/N- tertbutylacrylamide copolymer surfaces with changes in surface structure using contact angle measurements, molecular simulations, and Raman spectroscopy," Chemistry of Materials, vol. 17, no. 15, pp. 3889-3898, 2005.

[21] S. Lindman, I. Lynch, E. Thulin, H. Nilsson, K. A. Dawson, and S. Linse, "Systematic investigation of the thermodynamics of HSA adsorption to $\mathrm{N}$-iso-propylacrylamide/ $\mathrm{N}$-tertbutylacrylamide copolymer nanoparticles. Effects of particle size and hydrophobicity," Nano Letters, vol. 7, no. 4, pp. 914920, 2007.

[22] E. Farkas, R. Zelkó, Z. Németh, J. Pálinkás, S. Marton, and I. Rácz, "The effect of liquid crystalline structure on chlorhexidine diacetate release," International Journal of Pharmaceutics, vol. 193, no. 2, pp. 239-245, 2000.

[23] R. Pelton, “Temperature-sensitive aqueous microgels," Advances in Colloid and Interface Science, vol. 85, no. 1, pp. 1-33, 2000. 
[24] B. R. Saunders and B. Vincent, "Microgel particles as model colloids: theory, properties and applications," Advances in Colloid and Interface Science, vol. 80, no. 1, pp. 1-25, 1999.

[25] B. Sun, Y. Lin, and P. Wu, "Structure analysis of poly(N-isopropylacrylamide) using near-infrared spectroscopy and generalized two-dimensional correlation infrared spectroscopy," Applied Spectroscopy, vol. 61, no. 7, pp. 765-771, 2007.

[26] B. Sun, Y. Lin, P. Wu, and H. W. Siesler, "A FTIR and 2DIR spectroscopic study on the microdynamics phase separation mechanism of the poly( $\mathrm{N}$-isopropylacrylamide) aqueous solution," Macromolecules, vol. 41, no. 4, pp. 1512-1520, 2008.

[27] D. Duracher, A. Elaïssari, and C. Pichot, "Characterization of cross-linked poly(N-isopropylmethacrylamide) microgel latexes," Colloid and Polymer Science, vol. 277, no. 10, pp. 905913, 1999.

[28] J. M. Cornejo-Bravo, M. E. Flores-Guillen, E. Lugo-Medina, and A. Licea-Claverie, "Drug release from complexes with a series of poly (carboxyalkyl methacrylates), a new class of weak polyelectrolytes," International Journal of Pharmaceutics, vol. 305, no. 1-2, pp. 52-60, 2005.

[29] L. C. McCoy, C. J. Wehler, S. E. Rich, R. I. Garcia, D. R. Miller, and J. A. Jones, "Adverse events associated with chlorhexidine use: results from the Department of Veterans Affairs Dental Diabetes Study," Journal of the American Dental Association, vol. 139, no. 2, pp. 178-183, 2008.

[30] P. Axelsson and J. Lindhe, "Efficacy of mouthrinses in inhibiting dental plaque and gingivitis in man," Journal of Clinical Periodontology, vol. 14, no. 4, pp. 205-212, 1987.

[31] H. Jarvinen, J. Tenovuo, and P. Huovinen, "In vitro susceptibility of Streptococcus mutans to chlorhexidine and six other antimicrobial agents," Antimicrobial Agents and Chemotherapy, vol. 37, no. 5, pp. 1158-1159, 1993.

[32] G. Westergren and C. G. Emilson, "In vitro development of chlorhexidine resistance in Streptococcus sanguis and its transmissibility by genetic transformation," Scandinavian Journal of Dental Research, vol. 88, no. 3, pp. 236-243, 1980.

[33] A. S. Wadajkar, B. Koppolu, M. Rahimi, and K. T. Nguyen, "Cytotoxic evaluation of N-isopropylacrylamide monomers and temperature-sensitive poly(N-isopropylacrylamide) nanoparticles," Journal of Nanoparticle Research, vol. 11, no. 6, pp. 1375-1382, 2009. 

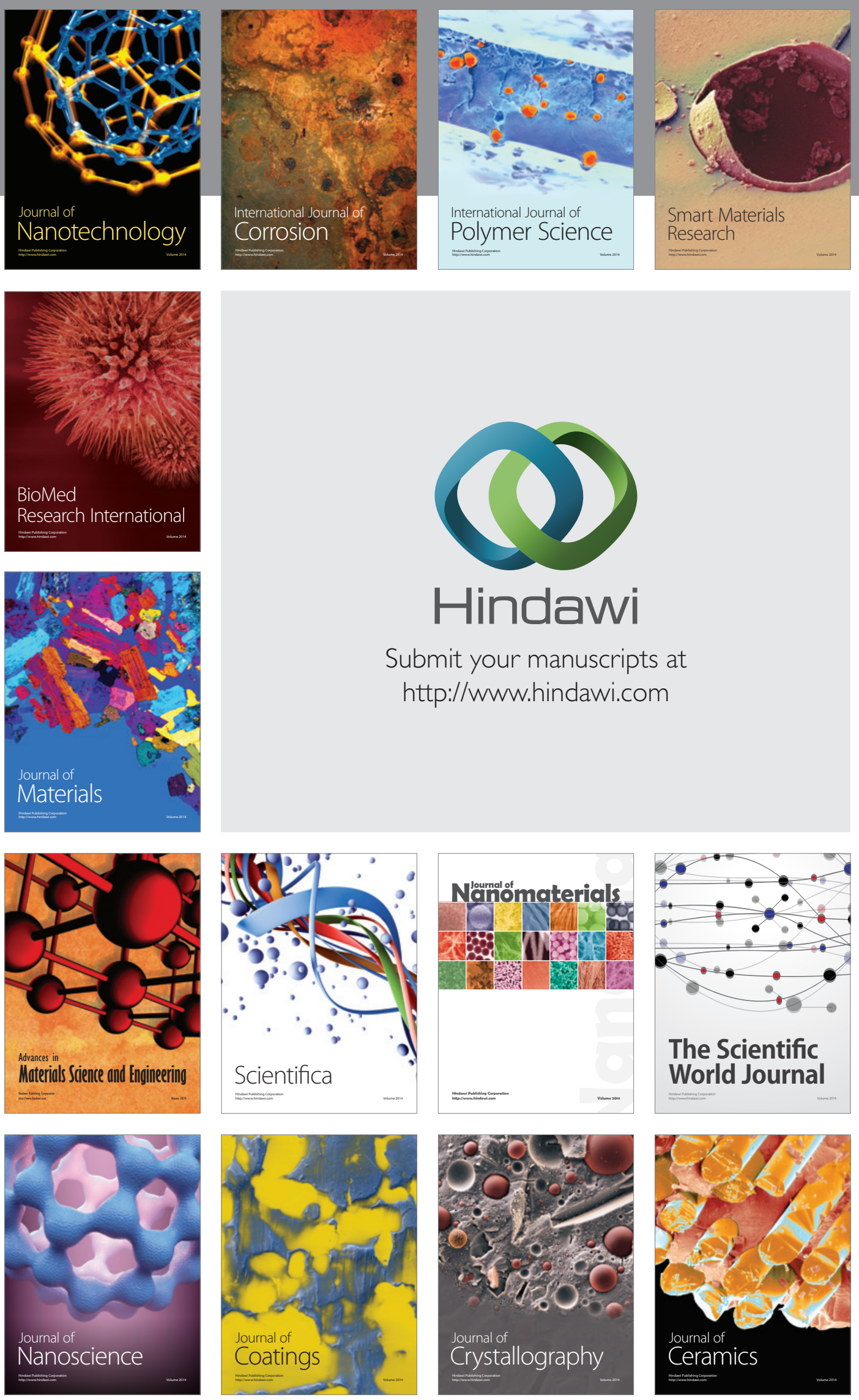

The Scientific World Journal

Submit your manuscripts at

http://www.hindawi.com

\section{World Journal}

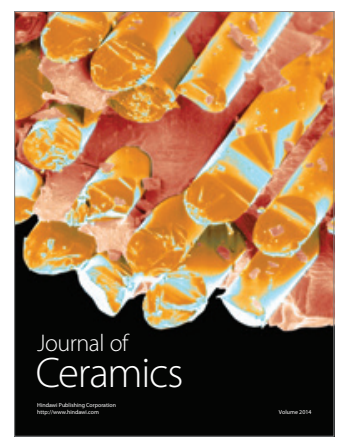

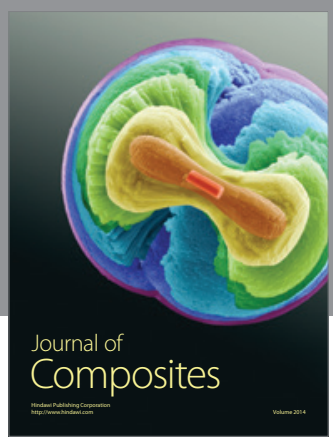
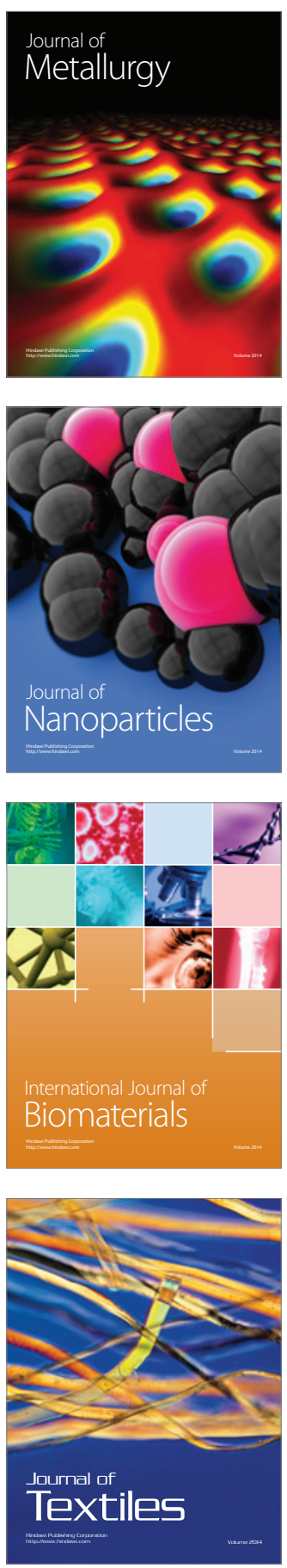